\title{
Variation in treatment of blunt splenic injury in Dutch academic trauma centres
}

Olthof DC ${ }^{1}$, MSc., d.c.olthof@amc.nl

Luitse JSK ${ }^{1}$, MD, j.s.luitse@ amc.nl.

de Rooij PP ${ }^{2}$, MD, p.derooij@erasmusmc.nl

Leenen LPH ${ }^{3}$, MD, PhD, Prof., 1.p.h.leenen@umcutrecht.nl

Wendt KW ${ }^{4}, \mathrm{MD}, \mathrm{PhD}$, k.w.wendt@umcg.nl

Bloemers FW ${ }^{5}$, MD, PhD, fw.bloemers@ vumc.nl

Corresponding author: Goslings JC ${ }^{1}$, MD, PhD, Prof. j.c.goslings@ amc.nl, Meibergdreef 9, 1105 AZ Amsterdam, the Netherlands. T: + 31 20-5666019 |F: +31 20-6914858 |E:

1: Trauma Unit Department of Surgery, Academic Medical Centre, Amsterdam, The Netherlands

2: Trauma Research Unit Department of Surgery, Erasmus MC, University Medical Centre Rotterdam, Rotterdam, The Netherlands

3: Department of Surgery, University Medical Centre Utrecht, The Netherlands

4: Department of Surgery, University Medical Centre Groningen, Groningen, The Netherlands

5: Department of Surgery, VU University Medical Centre, The Netherlands

\section{Author contributions}

Study conception and design: Olthof, Luitse, Goslings

Acquisition of data: Olthof, de Rooij

Analysis and interpretation of data: Olthof, Luitse, Bloemers, Leenen, de Rooij, Wendt,

Goslings

Drafting of manuscript: Olthof

Critical revision: Olthof, Luitse, Bloemers, Leenen, de Rooij, Wendt, Goslings

\section{Author diclosures}

The authors report no proprietary or commercial interest in any product mentioned or concept discussed in this article. 


\begin{abstract}
Introduction: The incidence of splenectomy following trauma is institutionally dependent and varies from $18 \%$ to as much as $40 \%$. This is important because variation in management influences splenic salvage. The aim of this study was to investigate whether differences exist between Dutch level 1 trauma centres with respect to the treatment of these injuries, and if variation in treatment was related to splenic salvage, spleen related re-interventions and mortality.
\end{abstract}

Methods: Consecutive adult patients who were admitted between January 2009 and December 2012 to five academic level 1 trauma centres were identified. Multinomial logistic regression was used to measure the influence of hospital on treatment strategy, controlling for haemodynamic (HD) instability upon admission, high grade (AAST 3-5) splenic injury, and injury severity score. Binary logistic regression was used to quantify differences between hospitals in splenic salvage rate.

Results: 253 patients were included: 149 (59\%) were observed, 57 (23\%) were treated with Splenic Artery Embolisation (SAE) and 47(19\%) were operated. The observation rate was comparable in all hospitals. SAE and surgery rates varied from $9 \%$ to $32 \%$ and $8 \%$ to $28 \%$, respectively. After adjustment the odds of operative management were significantly higher in one hospital compared to the reference hospital (adjusted OR 4.98 (1.02 - 24.44). The odds of splenic salvage were significantly lower in another hospital compared to the reference hospital (adjusted OR 0.20 (0.03-1.32).

Conclusion: Although observation rates were comparable between the academic trauma centres, embolisation and surgery rates varied. A nearly 5-fold increase in the odds of operative management was observed in one hospital, and another hospital had significantly lower odds of splenic salvage. The development of a national guideline is recommended to minimalize splenectomy after trauma.

Keywords: blunt splenic injury; treatment variation; splenic salvage 


\subsection{Introduction}

The prevalence of intra-abdominal injury among patients who present to the emergency department with Blunt Abdominal Trauma (BAT) is approximately 13 percent [1]. The spleen is one of the most commonly injured organs after the occurrence of blunt trauma.

Since the 1990s, angio-embolisation has been used as an alternative to operative management in the treatment of blunt abdominal injury. Currently, non-operative management (NOM) involving close observation of the patient, supplemented with splenic artery embolisation (SAE) when necessary, has become the standard treatment for haemodynamically stable patients. Recent reports, have however revealed that there is variation in treatment of splenic injury [2-5]. A recent report from the National Trauma Data Bank (NTDB), for example, indicated that the incidence of splenectomy at a number of institutions could vary from $18 \%$ to as much as $40 \%$, and that this percentage is highly dependent on the definition that is applied to NOM [4]. This is important because it has been shown that variation in management can influence splenic salvage [5;6]. Banerjee et al. compared trauma centres with high rates (defined as $>10 \%)$ of SAE to centres with low rates $(<10 \%)$ and found that patients treated at high SAE volume centres were less likely to undergo splenectomy, both after observation and SAE [6].

As there is no national protocol in the Netherlands which stipulates preferred treatment strategy, variation in management is likely to exist. In this study we investigated whether there were any differences in the treatment of blunt splenic injuries at five (academic) level 1 trauma centres. We also related these variations in treatment to splenic salvage, spleen related re-interventions and mortality rates.

\subsection{Methods}

In this retrospective observational study, the Trauma Registry databases of five academic level 1 hospitals (study period 2009 - 2012) were consulted to identify patients with blunt splenic injury. Abbreviated Injury Scale (AIS) diagnosis codes starting with 5442 were employed to retrieve the eligible patients from the registries. The study population consisted of adult (aged 16 years or older) patients.

\subsubsection{Data Collection}

The following data were collected: age, gender, Systolic Blood Pressure (SBP), haemoglobin level and Glasgow Coma Scale (GCS) upon admission, endotracheal intubation (yes or no), 
imaging for diagnosing splenic injury, grade of splenic injury (graded according to the American Association for the Surgery of Trauma[7]), the presence of a splenic contrast extravasation at i.v. contrast enhanced abdominal CT scanning, associated injuries and Injury Severity Score (ISS), treatment type (observation, SAE or operative treatment), complications, hospital - and intensive care (IC) unit length of stay (LOS), splenic salvage (spleen in situ at discharge), the need for and type of re-intervention, readmission (if yes) and mortality. AAST grading was assessed from the original radiology report when available. We graded patients with a diagnosis 'Contusion (hematoma) No Further Specified (NFS)' and 'Spleen NFS' as grade 1 injury, 'Laceration NFS' and 'Rupture NFS' as grade 2 injury for data analysis. In the patients who only received a Focussed Assessment for Sonography with Trauma (FAST) we could not assess the grade of splenic injury nor whether a contrast extravasation (variables scored as unknown) was present. The associated intra-abdominal injuries were further specified into injuries with an operation indication (such as perforation of a hollow organ, dissection of a major abdominal vessel or a diaphragmatic rupture) and grade $\geq 3$ or higher solid organ injury since associated injuries might influence the choice to perform surgery.

\subsubsection{Trauma Setting \& Definitions}

In Level 1 trauma centers trauma patients with all types of injuries can be treated. In Level 2 no neurosurgical interventions can be performed and in Level 3 only patients with non-lifethreatening injuries can be treated.

Initial treatment strategy was defined as the first documented treatment strategy for the splenic injury. The operative management group consists of the patients in whom a splenectomy was performed as well as the patients in whom spleen preserving surgery was applied. Failure of treatment was defined as the need for a splenic (re)intervention: SAE or splenic surgery for patients who were initially selected for observation, re-SAE or splenic surgery for patients who were initially embolized, splenic re-operation for patients initially treated with spleen conserving surgery or a re-operation after initial splenectomy. Reinterventions performed for other abdominal injuries were not counted for the spleen-related re-interventions. Complications were defined as all complications during admission, including the spleen related complications.

The vital signs values that were used are the first values measured upon arrival at the emergency department. For patients that were transferred, the values (if known) and treatment strategy (if performed) in the hospital of initial assessment were employed. Haemodynamic 
(HD) instability was defined as a systolic blood pressure $<90 \mathrm{mmHg}$. Patients were analysed in the unstable group if splenic injury was the documented cause of the HD instability or if there was a reasonable to strong assumption that splenic injury was the cause of instability. All five hospitals have acceptable distance and transport times to the angiography suite as well as 24/7 availability of a skilled interventional radiologist and availability of an operation theater and a trauma surgeon. The five hospitals were anonymised and coded as A, B, C, D, E.

\subsubsection{Statistical Analysis}

Statistical analysis was performed using IBM SPSS ${ }^{\circledR}$ software package version 20 (Inc., Chicago, IL, USA). Categorical data were expressed as number (percentage) and continuous data as median (p25-p75). Multinomial logistic regression was used to measure the influence of hospital on treatment strategy, both unadjusted and controlling for haemodynamic instability upon admission, high grade splenic injury (AAST grade 3-5 splenic injury) and ISS. The chi-squared test was used to compare splenic salvage, spleen related re-interventions and mortality between hospitals. Because splenic salvage was one of the most important outcome measures, we additionally performed binary logistic regression to quantify differences between hospitals in splenic salvage rate, controlling for haemodynamic status upon admission, high grade splenic injury, ISS and treatment strategy. Hospital A was set as reference hospital in regression analysis since it is the hospital with the highest rate of splenic salvage. Odds ratios were reported with their $95 \%$ confidence interval.

\subsection{Results}

Two hundred ninety eight patients were eligible for inclusion during the study period. Patients who died in the Trauma Room before treatment could be performed $(n=12)$, transferred patients in whom insufficient information was available about initial work-up $(n=21)$ or patients in whom splenic injury was coded but not described in the medical chart or radiology reports $(n=11)$ were excluded. In addition, one patient in whom splenic injury was diagnosed upon CT-scanning after an emergency laparotomy $(n=1)$, was excluded. The study cohort consisted of 253 patients. Patient characteristics are presented in Table 1.

\subsubsection{Treatment strategy}

Initial treatment strategy for each of the hospitals over the total study period is depicted in Figure 1. A total of 149 patients (59\%) were observed, 57 (23\%) patients were treated with 
SAE and 47 patients (19\%) were treated surgically. The observation rate was comparable between hospitals (Figure 1). In hospital A, a high embolisation rate (32\%) and low operation rate $(8 \%)$ was employed. In hospital $\mathrm{C}$, the opposite was observed (5 of the $54(9 \%)$ patients were embolized and 15 (28\%) patients were initially operated). In hospital D and E an equal amount of patients was embolized as was operated (7 vs. 7 in hospital D and 13 vs. 13 in hospital E).

\subsubsection{Variation in treatment}

The results of multinomial logistic regression are presented in Table 2. The upper part of Table 2 shows that the unadjusted odds of being treated with embolisation was significantly lower in hospital $\mathrm{C}$ compared to the chosen reference hospital (OR $0.28(0.09-0.84))$. After adjusting for HD instability, high grade splenic injury and ISS, no differences were observed between hospitals with regard to embolisation. The odds of operative management were significantly higher in hospital C compared to the reference hospital (adjusted OR 4.98 (1.02 $-24.44))$.

\subsubsection{Outcome measures}

Outcome measures are depicted in Table 3. Splenic salvage rates differed statistically significant between the hospitals (range 71 to $94 \%, p=0.03$ ). Results of binary logistic regression analysis are displayed in Table 4. After correction for HD instability, high grade splenic injury, ISS, and treatment strategy, the odds of splenic salvage were significantly lower for hospital E compared to hospital A (adjusted OR $0.14(0.02-0.87)$ ).

The percentage of spleen related re-interventions varied from 6 to 21 . The re-intervention rate for observation was $12 \%$ (18/149), $16 \%$ for SAE (9/57) and $6 \%$ for splenic surgery (3/47). The type of re-interventions in the patients who were initially treated surgically include after an initial operative attempt (packing of the abdomen), a splenectomy after an initial spleen preserving attempt and a re-laparotomy because of persistent abdominal bleeding. Twenty nine patients $(11 \%)$ died. None of these 29 patients had isolated splenic injury. Twelve of the twenty nine (41\%) patients were HD unstable because of their splenic injury and all but one unstable patients were initially operated $(n=11)$. A splenectomy was performed in 9 of the 11 operated patients (one patient died during transport to the operating room and in the other patient the splenic injury could be treated with a splenic mesh). The cause of death in the HD stable patients was traumatic brain injury in 10 patients, hypotension of unknown origin (no signs of abdominal or thoracic origin) in 1 patient, exsanguination 
from an aortaduodenal fistula as a consequence of dislocation of the endovascular prosthesis of the superior mesenteric artery in 1 patient, and unknown in 1 patient. Two patients died after they needed CPR during hospital admission. None of the deaths were classified as preventable or could be attributed to non-operative management.

\subsection{Discussion}

Although observation rates between academic trauma centres were comparable (initial attempt in approximately $60 \%$ of the patients with blunt splenic injury), embolisation and surgery rates varied between five level 1 academic trauma centres. Even in a relatively small country like the Netherlands ( $\approx 16.5$ million inhabitants), variation exists in treatment of blunt splenic injuries. After adjusting for hemodynamic instability, high grade splenic injury and injury severity score, a nearly 5-fold increase in the odds of operative management was observed in one hospital.

Variation in the treatment of splenic injury is important since it has been shown to influence splenic salvage [5;6]. After correction for HD instability, high grade splenic injury and ISS (all being factors predicting the need for initial operation) another hospital had significantly lower odds of splenic salvage compared to our reference hospital. Whenever possible, a splenectomy should be avoided in order to prevent patients from having a lifelong higher risk of overwhelming post-splenectomy infection and the need for (repeat) immunizations and early use of antibiotics.

Substantial variation in splenectomy rates, depending on hospital type, was previously demonstrated, in a large nationwide study in the United States [8]. Studies in the paediatric population have showed treatment variation in relation to urban versus rural environment, trauma volume, and status [9;10]. These factors did not differ between the hospitals in our study: all were situated in a rural environment; all have teaching status and have a more or less comparable trauma volume. Also, we looked into further detail to the patients who were operated on for associated intra-abdominal injuries. It is imaginable that in those patients the surgeon might sooner decide to perform a splenectomy (instead of performing spleen preserving therapy or SAE), especially if high grade splenic injury is present or if a large amount of intra-abdominal blood is encountered. This does not seem to be the case in our cohort. The differences we found are therefore probably due to true differences in treatment strategy between hospitals and might be related to the lack of a national guideline.

The fact that the hospital with the highest operation rate was not identified to be significantly associated with lower splenic salvage is probably explained by the fact that some of the 
operated patients underwent spleen preserving surgery.

Optimal treatment selection of patients with blunt splenic injury is a continuing matter of research. At all times, efforts should be made to preserve the spleen, if possible (e.g., HD unstable patients not responding to resuscitation or patients with associated injuries requiring a laparotomy should directly be brought to the operation theatre). In order to do so, operative management should be avoided since the odds of splenic salvage are significantly lower compared to observation (Table 4). However, if an operation is indicated it should not be delayed since it is known from literature that the failure of nonoperative management (defined as the need for a laparotomy after an initial attempt of observation) has consequences beyond delay to operation. Namely, the risk of mortality from abdominal injuries [11]. In addition, increased length of hospital stay has been reported in patients who require a laparotomy after failure of NOM compared to patients who were initially operated [12]. Even if a number of patients who fail observation can be successfully treated with a second non-operative treatment modality (12 out of 18 in our cohort), we should not underestimate the increased length of hospital stay with the possible risk of complications (e.g. infection).

In patients who are injured to several body parts or patients with vascular injuries that are difficult to assess (i.e. the pelvis), however, initial surgical treatment might be preferred over embolisation. Other drawbacks of non-operative management include the risk of a delayed splenic rupture, a re-bleed, and the fact that visual inspection of abdominal contents is not possible. Foremost, from a patient's and physician's perspective, it is desirable that the initial treatment strategy, being non-operative management (embolisation) or surgery, is successful and that the life threatening haemorrhage is controlled as quickly as possible according to the principles of Advanced Trauma Life Support [13].

This study was limited by the relatively small sample size. Fifteen percent of the patients were excluded from analysis, mainly because they died before (splenic) treatment could be initiated or because they were transferred from another hospital and insufficient information about initial work-up was available. Larger numbers would have resulted in greater power to detect differences between hospitals and to correct for more variables in the regression model (e.g., age and associated abdominal injuries). Another limitation of the study, related to its retrospective design, is that the exact reasons for observing a patient, proceeding to the angiosuite or to the operating room could not always be retrieved from the medical file(s). Lastly, only a selection of all the 11 Dutch level 1 trauma centers participated in the study. It is interesting to repeat this analysis in a representative sample of level 1, level 2 and level 3 centres in the Netherlands, preferably with a prospective study design, and to initiate 
preparations for the development of a national guideline, depending on the results of that study.

\subsection{Conclusion}

Although observation rates were comparable between the five academic trauma centres, embolisation and surgery rates varied. A nearly 5-fold increase in the odds of operative management was observed in one hospital and another hospital had significantly lower odds of splenic salvage. 


\section{Reference List}

[1] Nishijima DK, Simel DL, Wisner DH, Holmes JF. Does this adult patient have a blunt intra-abdominal injury? JAMA 2012; 307(14):1517-1527.

[2] Hamlat CA, Arbabi S, Koepsell TD, Maier RV, Jurkovich GJ, Rivara FP. National variation in outcomes and costs for splenic injury and the impact of trauma systems: a population-based cohort study. Ann Surg 2012; 255(1):165-170.

[3] Zarzaur BL, Croce MA, Fabian TC. Variation in the use of urgent splenectomy after blunt splenic injury in adults. $J$ Trauma 2011; 71(5):1333-1339.

[4] Jeremitsky E, Smith RS, Ong AW. Starting the clock: defining nonoperative management of blunt splenic injury by time. Am J Surg 2013; 205(3):298-301.

[5] Moore FA, Davis JW, Moore EE, Jr., Cocanour CS, West MA, McIntyre RC, Jr. Western Trauma Association (WTA) critical decisions in trauma: management of adult blunt splenic trauma. J Trauma 2008; 65(5):1007-1011.

[6] Banerjee A, Duane TM, Wilson SP, Haney S, O'Neill PJ, Evans HL et al. Trauma center variation in splenic artery embolization and spleen salvage: a multicenter analysis. J Trauma Acute Care Surg 2013; 75(1):69-74.

[7] Moore EE, Cogbill TH, Jurkovich GJ, Shackford SR, Malangoni MA, Champion HR. Organ injury scaling: spleen and liver (1994 revision). J Trauma 1995; 38(3):323-324.

[8] Todd SR, Arthur M, Newgard C, Hedges JR, Mullins RJ. Hospital factors associated with splenectomy for splenic injury: a national perspective. J Trauma 2004; 57(5):1065-1071.

[9] Mooney DP, Rothstein DH, Forbes PW. Variation in the management of pediatric splenic injuries in the United States. J Trauma 2006; 61(2):330-333.

[10] Haan JM, Biffl W, Knudson MM, Davis KA, Oka T, Majercik S et al. Splenic embolization revisited: a multicenter review. J Trauma 2004; 56(3):542-547. 
[11] Peitzman AB, Harbrecht BG, Rivera L, Heil B. Failure of observation of blunt splenic injury in adults: variability in practice and adverse consequences. J Am Coll Surg 2005; 201(2):179-187.

[12] Harbrecht BG, Zenati MS, Ochoa JB, Townsend RN, Puyana JC, Wilson MA et al. Management of adult blunt splenic injuries: comparison between level I and level II trauma centers. J Am Coll Surg 2004; 198(2):232-239.

[13] American College of Surgeons Committee on Trauma (2014) Advanced trauma life support programs for doctors, $7^{\text {th }}$ edn. American College of Surgeons, Chicago, 
Table 1. Patient characteristics per hospital

\begin{tabular}{|c|c|c|c|c|c|}
\hline & $\begin{array}{c}\text { Hospital A } \\
\mathrm{n}=50\end{array}$ & $\begin{array}{c}\text { Hospital B } \\
\mathrm{n}=51\end{array}$ & $\begin{array}{c}\text { Hospital C } \\
\mathrm{n}=54\end{array}$ & $\begin{array}{c}\text { Hospital D } \\
\mathrm{n}=43\end{array}$ & $\begin{array}{c}\text { Hospital E } \\
\mathrm{n}=55\end{array}$ \\
\hline Age (years) & $30(23-55)$ & $30(21-51)$ & $31(19-52)$ & $36(21-50)$ & $33(23-43)$ \\
\hline Male gender & $38(76)$ & $36(71)$ & $46(85)$ & $32(74)$ & $35(64)$ \\
\hline \multicolumn{6}{|l|}{$\mathrm{SBP}(\mathrm{mmHg})$} \\
\hline $1-49$ & $1(2)$ & - & $1(2)$ & $1(2)$ & $1(2)$ \\
\hline $50-75$ & - & $2(4)$ & $9(17)$ & $4(9)$ & $5(9)$ \\
\hline $76-89$ & $2(4)$ & $2(4)$ & $2(4)$ & $7(16)$ & $5(9)$ \\
\hline$>89$ & $47(94)$ & $47(92)$ & $42(78)$ & $31(72)$ & $44(80)$ \\
\hline GCS on admission & $14(4-15)$ & $15(14-15)$ & $14(8-15)$ & $15(3-15)$ & $14(6-15)$ \\
\hline $\begin{array}{l}\text { Hemoglobin level on } \\
\text { admission }(\mathrm{g} / \mathrm{dL})\end{array}$ & $\begin{array}{c}11.4(9.4- \\
13.2)\end{array}$ & $\begin{array}{c}11.7(9.6- \\
13.8)\end{array}$ & $\begin{array}{c}13.7(11.7- \\
14.3)\end{array}$ & $\begin{array}{c}10.9(8.9- \\
12.7)\end{array}$ & $\begin{array}{c}12.4(11.0- \\
13.8)\end{array}$ \\
\hline ISS (points) & $29(20-42)$ & $22(16-36)$ & $26(17-39)$ & $29(17-41)$ & $29(22-45)$ \\
\hline \multicolumn{6}{|l|}{$\operatorname{Diagnostics}^{\dagger}$} \\
\hline FAST and CT-scan & $37(74)$ & $46(90)$ & $8(15)$ & $39(91)$ & $47(86)$ \\
\hline Only FAST & $2(4)$ & $2(4)$ & $8(15)$ & $4(8)$ & $6(11)$ \\
\hline Only CT-scan & $10(20)$ & $2(4)$ & $37(69)$ & - & $2(4)$ \\
\hline \multicolumn{6}{|l|}{ Isolated (splenic) } \\
\hline injury & $4(8)$ & $10(20)$ & $3(6)$ & $6(14)$ & $1(2)$ \\
\hline Associated IA injury & $24(48 \%)$ & $12(24 \%)$ & $25(49 \%)$ & $21(49 \%)$ & $29(53 \%)$ \\
\hline Operation indication & 1 & 1 & 4 & 2 & 3 \\
\hline$\geq$ Grade 3 SOI & 5 & 4 & 3 & 5 & 9 \\
\hline Grade of splenic injury & $3(1-4)$ & $3(2-4)$ & $2(1-3)$ & $2(2-3)$ & $3(2-4)$ \\
\hline $\begin{array}{l}\text { Contrast } \\
\text { extravasation }\end{array}$ & $18(36)$ & $21(41)$ & $5(9)$ & $18(42)$ & $22(40)$ \\
\hline Hospital LOS* & $8(5-24)$ & $14(10-24)$ & $10.5(4-18)$ & $16(10-34)$ & $15(8-36)$ \\
\hline IC LOS & $3(0-6)$ & $3(2-5)$ & $0.5(1-3)$ & $4(2-9)$ & $2(0-10)$ \\
\hline $\begin{array}{l}\text { Data are expressed as numbe } \\
\text { Abbreviations: SBP, systolic } \\
\text { Severity Score; SOI: Solid O } \\
\text { †: Patient was referred and in } \\
\text { no imaging was performed } \\
\text { : Unknown in } 29 \text { patients }(2 \\
*: 12 \text { patients died within } 1 \mathrm{~d} \\
\text { \#: Operation indication was d } \\
\text { a diaphragmatic rupture }\end{array}$ & $\begin{array}{l}\text { (percentage) or } \\
\text { blood pressure; } \\
\text { gan Injury } \\
\text { ormation about } \\
\text { in the initial ph } \\
\text { in hospital A, } 5 \\
\text { ay after admissic } \\
\text { efined as: perfor }\end{array}$ & $\begin{array}{l}\text { median (p25-p7 } \\
\text { JCS, Glasgow C } \\
\text { nitial diagnostic } \\
\text { se (one patient } \\
\text { n hospital B, } 11 \\
\text { n thereby loweri } \\
\text { tion of a hollow }\end{array}$ & $\begin{array}{l}\text { oma Score; IA: } \\
\text { was missing (o } \\
\text { hospital C). } \\
\text { in hospital C, } 4 \\
\text { ng the total leng } \\
\text { organ, dissectio }\end{array}$ & $\begin{array}{l}\text { ntra-Abdominal } \\
\text { he patient in hos } \\
\text { h of hospital D, } 7 \text { i sta }\end{array}$ & $\begin{array}{l}\text { ISS, Injury } \\
\text { ital A and B) or } \\
\text { hospital E. } \\
\text { minal vessel or }\end{array}$ \\
\hline
\end{tabular}


Table 2. Results of multinomial logistic regression for variation in treatment strategy, unadjusted (upper part of Table) and adjusted (lower part of Table)

\begin{tabular}{|c|c|c|}
\hline \multicolumn{3}{|c|}{ Unadjusted OR } \\
\hline & Embolisation & Operative Management \\
\hline Hospital A (reference) & - & - \\
\hline Hospital B & $1.11(0.47-2.64)$ & $2.22(0.60-8.22)$ \\
\hline Hospital C & $0.28(0.09-0.84)$ & $3.31(0.99-11.07)$ \\
\hline Hospital D & $0.45(0.16-1.26)$ & $1.81(0.48-6.85)$ \\
\hline Hospital E & $0.84(0.34-2.05)$ & $3.36(0.98-11.52)$ \\
\hline \multicolumn{3}{|c|}{ Adjusted OR } \\
\hline & Embolisation & Operative Management \\
\hline HD instability & $5.80(1.33-25.33)$ & $42.35(9.33-192.21)$ \\
\hline High grade splenic injury & $11.68(4.83-28.28)$ & $9.37(2.75-31.97)$ \\
\hline ISS (points) & $1.02(0.99-1.05)$ & $1.08(1.05-1.12)$ \\
\hline \multicolumn{3}{|l|}{ Hospital } \\
\hline A (reference) & - & - \\
\hline $\mathrm{B}$ & $1.40(0.51-3.84)$ & $2.93(0.57-15.00)$ \\
\hline $\mathrm{C}$ & $0.42(0.12-1.45)$ & $4.98(1.02-24.44)$ \\
\hline $\mathrm{D}$ & $0.43(0.13-1.45)$ & $0.61(0.09-4.07)$ \\
\hline $\mathrm{E}$ & $0.66(0.24-1.84)$ & $1.60(0.33-7.79)$ \\
\hline
\end{tabular}

Observation was set as reference treatment strategy and hospital A was set as reference hospital (hospital with the highest splenic salvage rates). For example: the odds of being treated with embolisation relative to the odds of being treated with observation (reference) is 1.11 (95\% CI 0.47-2.64) in hospital B compared to hospital A. ${ }^{\ddagger}$ Splenic injury grade 3-5 according to the American Association for the Surgery of Trauma. Emboldened values are statistically significant. 
Table 3. Outcome measures for the five hospitals

\begin{tabular}{lccc}
\hline & $\begin{array}{c}\text { Splenic salvage } \\
(\text { at discharge })\end{array}$ & $\begin{array}{c}\text { Spleen related } \\
\mathbf{N}(\%)\end{array}$ & $\begin{array}{c}\text { Mortality } \\
\mathbf{N}(\%)\end{array}$ \\
\hline Hospital A $(\mathrm{n}=50)$ & $47(94 \%)$ & $6(12 \%)$ & $7(14 \%)$ \\
\hline Hospital B $(\mathrm{n}=51)$ & $42(82 \%)$ & $6(12 \%)^{*}$ & $3(6 \%)$ \\
\hline Hospital C $(\mathrm{n}=54)$ & $40(74 \%)$ & $3(6 \%)$ & $7(13 \%)$ \\
\hline Hospital D $(\mathrm{n}=43)$ & $36(84 \%)$ & $9(21 \%)^{*}$ & $6(14 \%)$ \\
\hline Hospital E $(\mathrm{n}=55)$ & $39(71 \%)$ & $6(11 \%)$ & $6(11 \%)$ \\
\hline P-value & $\mathbf{0 . 0 3}$ & 0.26 & 0.68 \\
\hline
\end{tabular}

Emboldened values are statistically significant $(\mathrm{P}$-value $<0.05)$

*: in one patient angiography was performed but the contrast extravasation could not be detected anymore during imaging. Therefore, the interventional radiologist refrained from treatment 
Table 4. Results of binary logistic regression analysis for splenic salvage

\begin{tabular}{lll}
\hline Variable & Splenic salvage (OR $(95 \%$ CI $))$ & P-value \\
\hline Treatment strategy* & & - \\
Observation & - & 0.56 \\
SAE & $0.66(0.16-2.78)$ & $<\mathbf{0 . 0 0 1}$ \\
Operative treatment & $\mathbf{0 . 0 1}(\mathbf{0 . 0 0 - 0 . 0 6})$ & \\
\hline Hospital* & & - \\
Hospital A (reference) & - & 0.17 \\
Hospital B & $0.26(0.04-1.78)$ & 0.09 \\
Hospital C & $0.20(0.03-1.32)$ & 0.15 \\
Hospital D & $0.22(0.03-1.74)$ & $\mathbf{0 . 0 4}$ \\
Hospital E & $\mathbf{0 . 1 4}(\mathbf{0 . 0 2}-\mathbf{0 . 8 7})$ &
\end{tabular}

* The effect of hospital on splenic salvage, controlled for haemodynamic instability, high grade splenic injury (AAST grade 3-5), ISS.

Abbreviations: SAE: Splenic Artery Embolisation

Observation was set as reference treatment strategy and Hospital A was set as reference hospital (hospital with the highest splenic salvage rates).

Emboldened values are statistically significant $(\mathrm{P}$-value $<0.05)$. 
Figure 1. Initial treatment strategy for each of the hospitals over the total study period

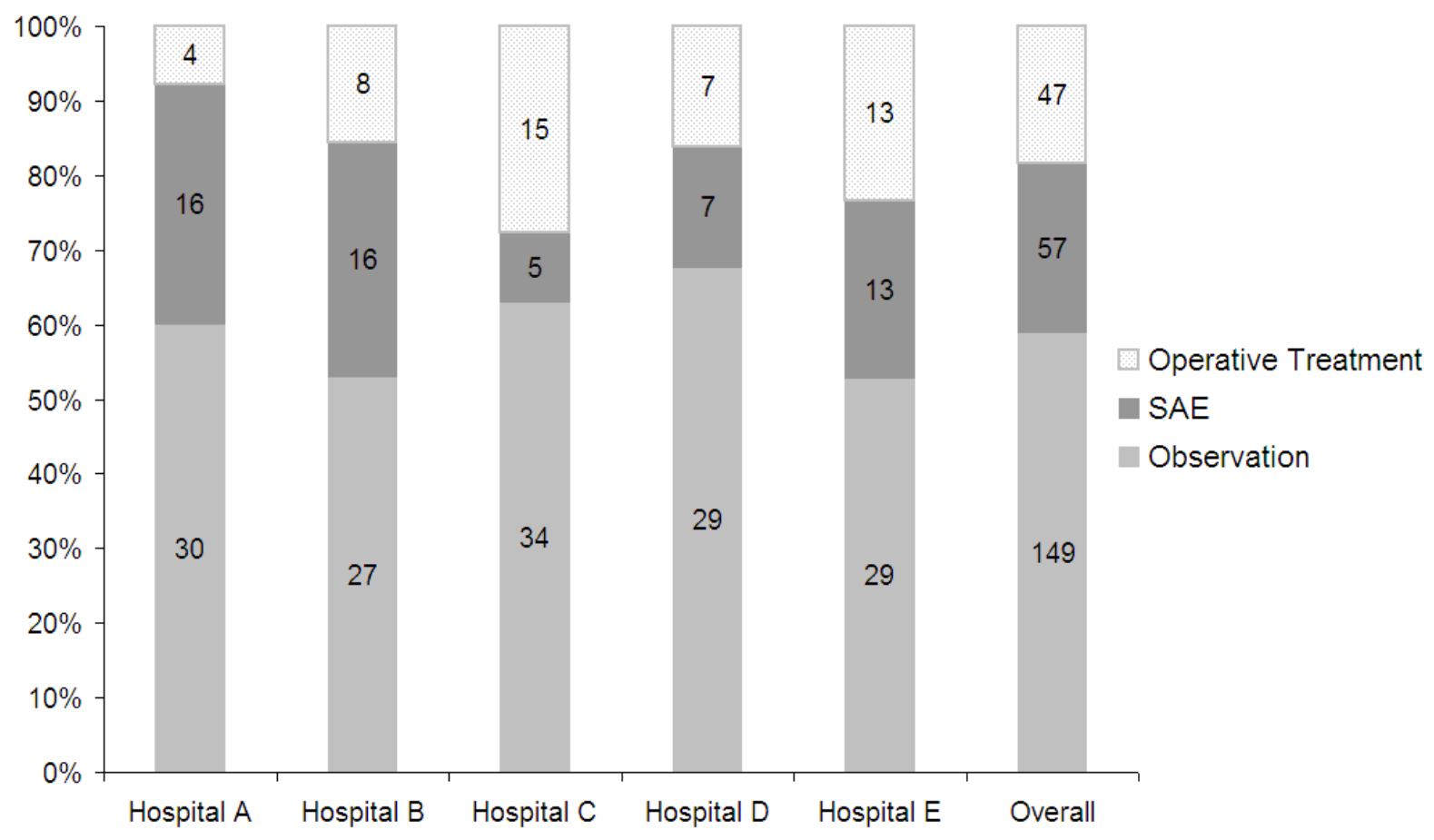

